\title{
Evaluating success of no-scalpel vasectomy by ligation and excision with fascial interposition in a large prospective study in Islamic Republic of Iran
}

\author{
H.R. Farrokh-Eslamlou, ${ }^{1}$ M. Eslami, ${ }^{1}$ I. Abdi-Rad ${ }^{1}$ and B. Eilkhanizadeh ${ }^{1}$
}

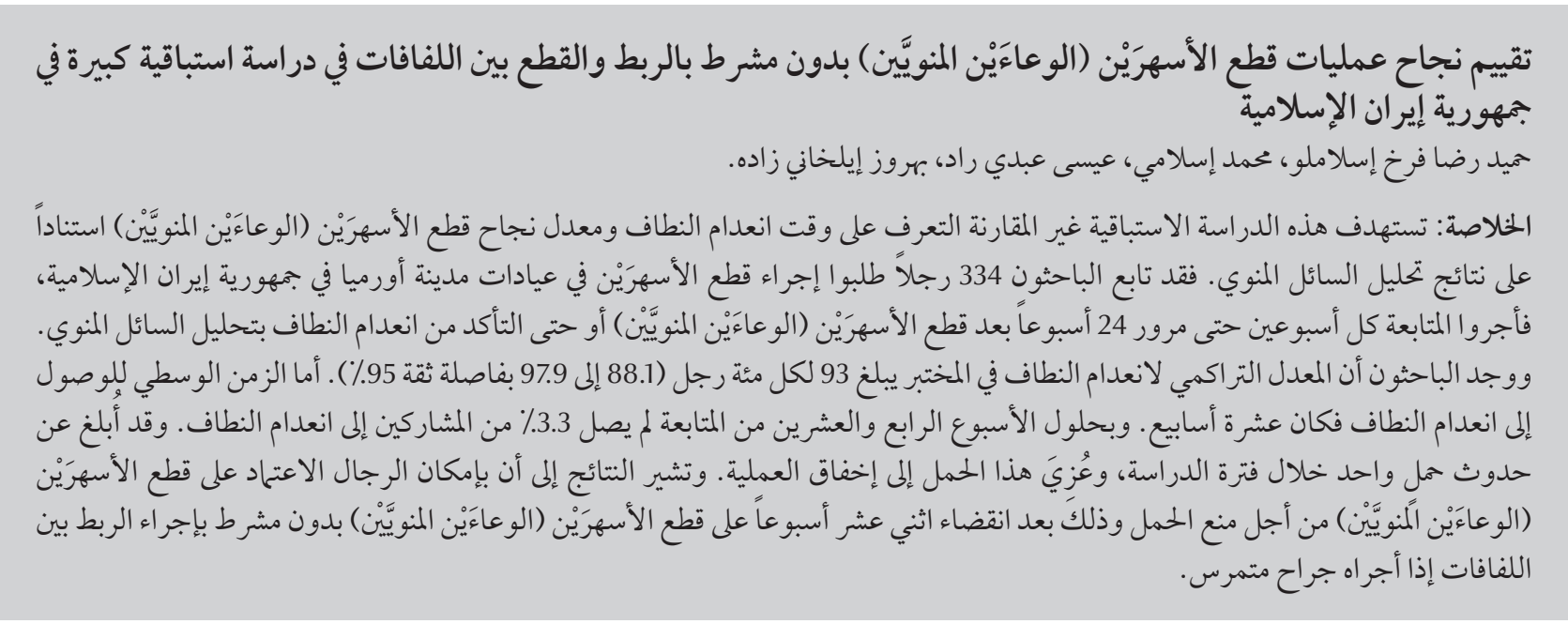

ABSTRACT The aims of this prospective, non-comparative study were to determine time to azoospermia and vasectomy success rate based on the results of semen analysis. A total of 334 men seeking vasectomy at a clinic in Urmia city, Islamic Republic of Iran were followed bi-weekly up to 24 weeks after vasectomy or until azoospermia was confirmed via semen analysis. The cumulative life table rate for azoospermia was 93/100 men (95\% Cl: 88.1 to 97.9). The median time to azoospermia was 10 weeks. By week 24 of follow-up, 3.3\% of participants had failed to achieve azoospermia. One pregnancy was reported during the study period and attributed to user failure. The results suggest that men can begin to rely on vasectomy for contraception 12 weeks after no-scalpel vasectomy using fascial interposition performed by an experienced surgeon.

Évaluation de l'efficacité de la vasectomie sans bistouri par ligature et excision avec interposition fasciale dans une étude prospective à grande échelle en République islamique d'Iran

RÉSUMÉ Les objectifs de la présente étude prospective et non comparative étaient de déterminer le temps jusqu'à l'azoospermie et le taux d'efficacité de la vasectomie mesurés par spermogramme. Au total, 334 hommes ayant subi une vasectomie dans un dispensaire de la ville d'Urmia (République islamique d'Iran) ont été suivis deux fois par semaine et jusqu'à 24 semaines après l'intervention ou jusqu'à la confirmation de l'azoospermie au moyen du spermogramme. Le taux cumulé pour l'azoospermie en utilisant la table de survie était de 93/100 hommes (IC à $95 \%$ : 88,1 à 97,9). Le temps médian jusqu'à l'azoospermie était de dix semaines. À la vingt-quatrième semaine de suivi, 3,3 \% des participants n'avaient pas obtenu une azoospermie. Une grossesse a été rapportée pendant la période de l'étude et imputée au comportement du patient. Les résultats suggèrent que les hommes peuvent commencer à compter sur l'efficacité de la vasectomie pour assurer leur contraception douze semaines après une intervention sans bistouri avec interposition fasciale réalisée par un chirurgien expérimenté. 


\section{Introduction}

Vasectomy is one of the safest family planning methods currently available and continues to be the most reliable form of male contraception worldwide. Over 45 million couples rely on vasectomy as their method of family planning [1]. Li Shunqiang of the Chongqing Family Planning Scientific Research Institute in China developed the noscalpel vasectomy (NSV) technique in 1974. Today, NSV is a popular family planning method in many countries as it eliminates concerns about incisions and has a low complication rate [2].

The success of vasectomy is confirmed by the demonstration of azoospermia (absence of sperm in the ejaculate). Even so, the desired endpoint of azoospermia is not achieved immediately after surgery and requires analysis of semen samples obtained at one or more clinic visits following the procedure. It is widely accepted in clinical practice that becoming azoospermic may take up to several months for most men because of sperm residing in the seminal vesicles and vas deferens upstream from the surgical incision [3]. The duration of the risk of pregnancy after a vasectomy varies greatly and is based on factors such as the time and/ or the number of ejaculations since vasectomy, sperm motility, sperm viability and the method of vas occlusion [4-7]. In the clinical setting, recommendations for semen analysis vary widely from 1 to 12 months [8]. In addition, since men are advised to use alternative contraception until azoospermia is confirmed, a long interval to the first follow-up visit may be problematic for some men, and those who do not demonstrate azoospermia must return for further visits, and therefore follow-up rates and compliance with semen testing protocols are often poor $[9,10]$. While counselling guidelines commonly used in vasectomy centres recommend that men can rely on vasectomy for contraception after 20 ejaculations or 2 months [11,12], in the Islamic Republic of Iran the recommended time interval is 3 months.

While many reports have been published on the time to azoospermia and the number of ejaculations required for achieving azoospermia following vasectomy, most of these are retrospective, descriptive studies, and interpreting the data are difficult $[13,14]$. One carefully controlled prospective study on the time and number of ejaculations to azoospermia after vasectomy by ligation and excision showed only 60/100 and $27.9 / 100$ of men were azoospermic by 12 weeks and 20 ejaculations, respectively [15]. This result is different from our experience at the national NSV training centre in the Islamic Republic of Iran after more than 9000 vasectomies using the NSV technique. We therefore conducted this large prospective study to examine the vasectomy success rate of NSV with fascial interposition, to determine the time to azoospermia and to evaluate adverse events associated with the method.

\section{Methods}

\section{Study design}

This was a prospective, non-comparative study of men followed for up to 24 weeks after vasectomy. The study was conducted from January 2007 to December 2008 at a reproductive health research centre in Urmia city, Islamic Republic of Iran.

\section{Sample}

As a routine of the clinic, all vasectomy clients together with their wives participated in family planning counselling to make sure that vasectomy was their informed choice. Men seeking vasectomy were asked if they would like to participate in the study, and then were screened for eligibility. Informed consent, a medical history, physical examination and a pre-vasectomy semen sample were obtained.
The eligibility criteria included: meeting the above-mentioned clinic criteria for vasectomy; being currently sexually active; willing to provide a semen specimen pre-vasectomy and biweekly up to 24 weeks after vasectomy or until azoospermia was confirmed; and freely consenting to participate in the study and sign an informed consent form. Exclusion criteria were: any acute or febrile illness; history of prior vasectomy or other scrotal surgery; clinical evidence of active sexually transmitted infection; a large varicocele or other scrotal mass; or taking any type of anabolic steroids.

Over the period of the study out of a total of 1622 men who underwent vasectomy at Urmia reproductive health research centre 380 (23.4\%) were compatible with the eligibility criteria and were enrolled in the study. Of the 380 enrolled men, 46 (12.1\%) never returned for follow-up after vasectomy and were therefore excluded from analysis. The study group therefore included 334 men who delivered at least 1 semen specimen after vasectomy.

\section{Vasectomy technique}

Vasectomy was performed using the no-scalpel technique following local infiltration with $2 \%$ epinephrine-free lidocaine. The vas deferens was ligated at 2 locations approximately $1.5 \mathrm{~cm}$ apart using 2 separate 2.0 silk ligatures and the segment of vas about $1 \mathrm{~cm}$ between the ligations was excised. Then fascial interposition was performed with the stump of the vasal prostatic end outside the fascial sheath and the stump of the testicular end inside the fascial sheath. The surgeons were master trainers in the technique, which ensured that standardized NSV with fascial interposition was used for all participants. Prior to the study the surgeons attended a meeting where the technique was standardized under the supervision of the developers of the NSV technique in China.

After vasectomy, participants were given instructions about the conduct of 
the study and asked to return bi-weekly until they were told that they could rely on vasectomy for contraception. A semen analysis was performed at postvasectomy follow-up visits. Men who did not attain azoospermia by 24 weeks after vasectomy were counselled regarding potential fertility and offered additional semen testing, another vasectomy or other contraceptives. At all follow-up visits, the investigators examined each patient for granuloma, haematoma, epididymitis or wound infection.

\section{Semen analysis procedures}

We performed semen analysis prevasectomy and then bi-weekly up to 24 weeks after vasectomy or until azoospermia was confirmed. Men who did not develop azoospermia at week 24 were followed up to week 34 to evaluate the delayed onset of azoospermia.

Participants produced semen samples at their home. Semen was examined for concentration and motility within 1 hour of collection using procedures based on World Health Organization guidelines [16] under the supervision of a pathologist who was expert in this procedure. Briefly, an aliquot of semen was examined by phase-contrast microscopy at high-power magnification $(x 400)$ to estimate sperm concentration. Appropriate dilutions of $1: 4$ to 1:20 depending on the estimated concentration were prepared using water to determine the concentration and using saline for motility subjective assessment ( $0 \%$ to $100 \%$ ). Concentration and motility were determined using an improved Neubauer haemocytometer. If no sperm were seen on the initial phase contrast examination, semen samples were centrifuged at $600 \mathrm{~g}$ for 15 minutes and then were examined as previously described.

\section{Analysis}

Men who delivered at least 1 postvasectomy sample were included in the analysis. Participants' baseline information (age, spouse's age and pre- vasectomy semen analysis measures) was summarized using mean, standard deviation (SD), minimum, maximum and median values. Vasectomy success, i.e. azoospermia, was defined as 2 consecutive semen specimens without sperm. The follow-up weeks were defined with a follow-up window of 14 (SD 7) days from the previous visit. For example, week 16 was defined as a visit $\geq 108$ days or $\leq 119$ days since the procedure. The presence of any motile sperm at week 24 or later in the semen specimen was used to define vasectomy failure.

In evaluating azoospermia and motility, 2 outcome measures were evaluated: the median number of weeks to azoospermia or zero motility; and the week in which the cumulative rate reached the maximum level observed during the study.

Changes over time were evaluated first for each outcome for all ages combined, followed by the results stratified by age. The time to azoospermia were calculated from the date of vasectomy to the date of the first specimen without sperm. The censor date for men not achieving azoospermia was the date of the lastsemen analysis. To calculate time to azoospermia, cumulated life-table probabilities were used. The results are reported at 12 weeks after vasectomy due to current counselling guidelines in the Islamic Republic of Iran.

\section{Results}

\section{Participants' characteristics}

Of the 334 men, 280 participants (83.8\%) demonstrated azoospermia according to the study definition; 11 participants $(3.3 \%)$ completed the study but did not achieve azoospermia by 24 weeks of follow-up and so were defined as vasectomy failure; 43 participants (12.9\%) discontinued the study prior to reaching azoospermia or reaching 24 weeks follow-up. Of the latter group 13 (3.9\%) discontinued for personal reasons and the other 30 $(9.0 \%)$ for unknown reasons, i.e. were lost to follow-up. The 43 men who had the potential to reach azoospermia but whose semen analysis status was unknown were included in all the analyses. No pregnancies related to vasectomy failure were reported.

\section{Baseline data}

The mean age of all study participants who delivered at least 1 sample for analysis after vasectomy was 40.9 years (range 26 to 76 years). The mean age of participants' wives was 34.4 years (range 22 to 53 years). All participants were married men, their spouse had most commonly used the combined oral contraceptive pill before the vasectomy (32.9\%) and most of them had 2 children (59.9\%).

The mean volume of ejaculate analysed in the pre-vasectomy semen specimen was $3.6 \mathrm{~mL}$ (range 0.6-6.7 $\mathrm{mL}$ ). The mean concentration of the sperm in the samples was 115 million/ $\mathrm{mL}$ (range 20-250 million/mL). The mean percentage of motile sperm was $66.6 \%$ (range $34 \%$ to $88 \%$ ).

\section{Time to azoospermia}

For the primary analysis, the cumulative survival curve of time to azoospermia estimated by the life table method was calculated (Figure 1). At the end of week 22, which was the last visit for determining azoospermia, the cumulative life table rate for azoospermia was 93/100 men [95\% confidence interval (CI): 88.1-97.9].

When time to azoospermia was examined by age group, younger men reached azoospermia at a faster rate (Figure 2). The cumulative life table rate for men under age 40 years reached its maximum of $95.5 / 100$ men ( $95 \% \mathrm{CI}$ : 90.4-00.6). At the age 40+ years, men reached the cumulative life table rate of 89/100 men (95\% CI: 82.5-95.5).

The median time to success (azoospermia) was 10 weeks for all participants. Among men under age 


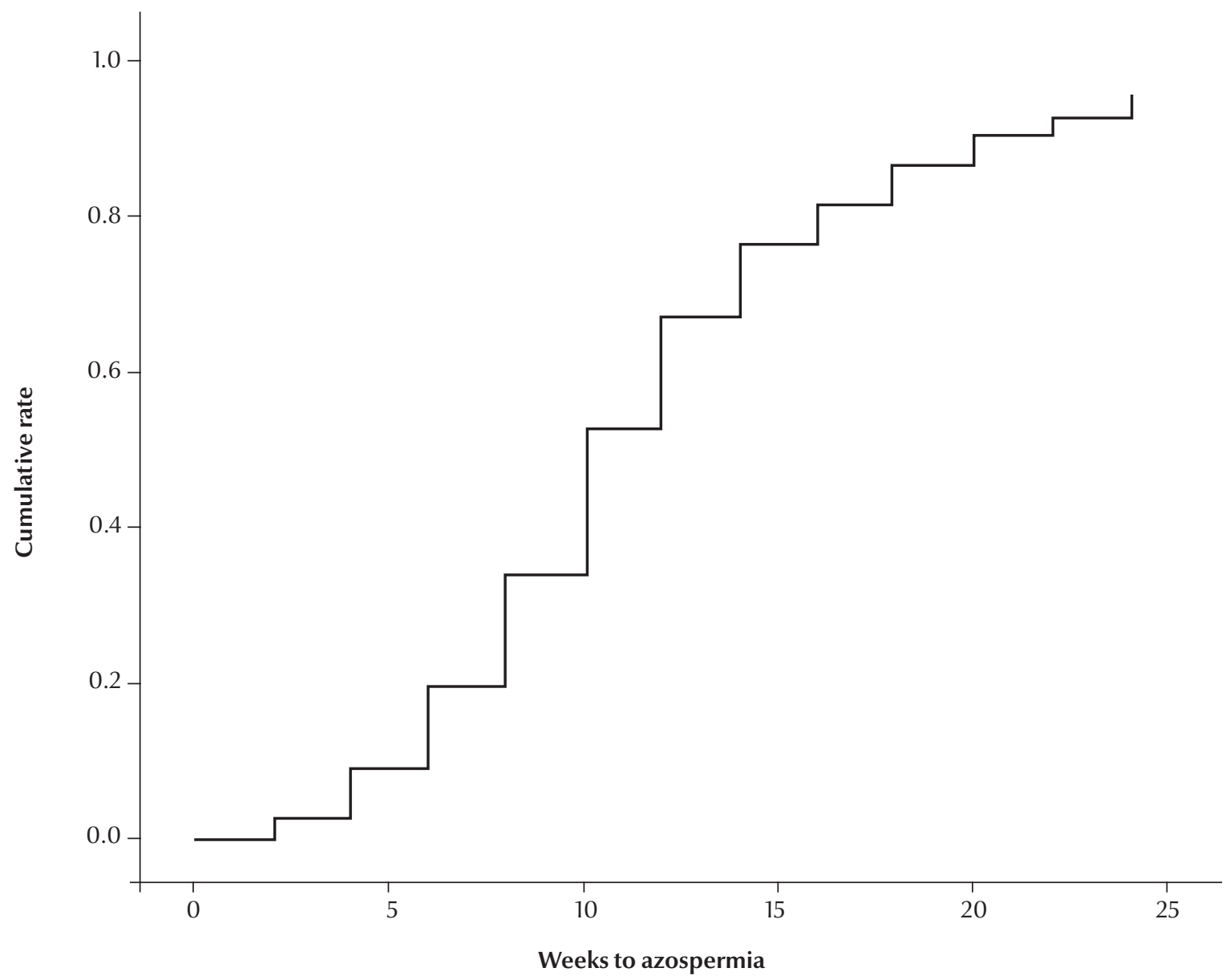

Figure 1 Gross cumulative life table probabilities for azoospermia after no-scalpel vasectomy by ligation and excision with fascial interposition $(n=334)$

40 years and men of age $40+$ years the median times to azoospermia were 8.5 and 11.5 weeks respectively.

The cumulative survival probability for azoospermia was 76.0/100 men (95\% CI: 69.6-82.4) at 12 weeks (Figure 1).

\section{Vasectomy failure based on semen analysis}

By week 24 of follow-up, 11 of the 334 participants (3.3\%) had not achieved azoospermia. All of these men accepted an offer of extended follow-up to 34 weeks. Ofthis group 4 participants ( $1.2 \%$ of the total) ended the 24 weeks followup with persistent low sperm concentrations (mean 25000 sperm/mL), but reached azoospermia at 34 weeks after vasectomy. Presumably these men had delayed onset success of vasectomy. The other 7 participants (2.1\% of total) had sperm concentrations greater than 2 million sperm/ mL (mean 25 million sperm $/ \mathrm{mL}$ ) with many motile sperm at 34 weeks. These men were considered to have vasectomy failure.

\section{Pregnancies}

One pregnancy was reported during the study period. This pregnancy occurred about 1 month after vasectomy, when the participant failed to use back-up family planning method as instructed until reaching azoospermia. The participant reported that prescribed condoms were not used during sexual intercourse. This was considered a user failure. Among the 8 men with vasectomy failures, 6 accepted an offer of repeat vasectomy.

\section{Adverse events}

At every post-vasectomy follow-up visit, all of the participants were clinically examined and were asked questions about probable adverse events. The most commonly reported adverse event was mild post-surgical local pain. Among 286 men (85.6\%) with data on post-surgical pain, 145 (50.7\%) reported taking no analgesics. 84 (29.4\%) reported analgesic use for a maximum of 3 days, 41 (14.3\%) for up to 1 week, and 16 (5.6\%) for more than 1 week.

Urogenital adverse events considered to be related to the procedure were: sperm granuloma in 27 (8.1\%), epididymitis or orchitis in $4(1.2 \%)$, scrotal pain or swelling in 6 cases (1.8\%) and haematomas in 5 (1.5\%). The haematomas were small and none required drainage. Infections occurred in 2 $(0.6 \%)$ of the cases. No serious adverse events were reported. 


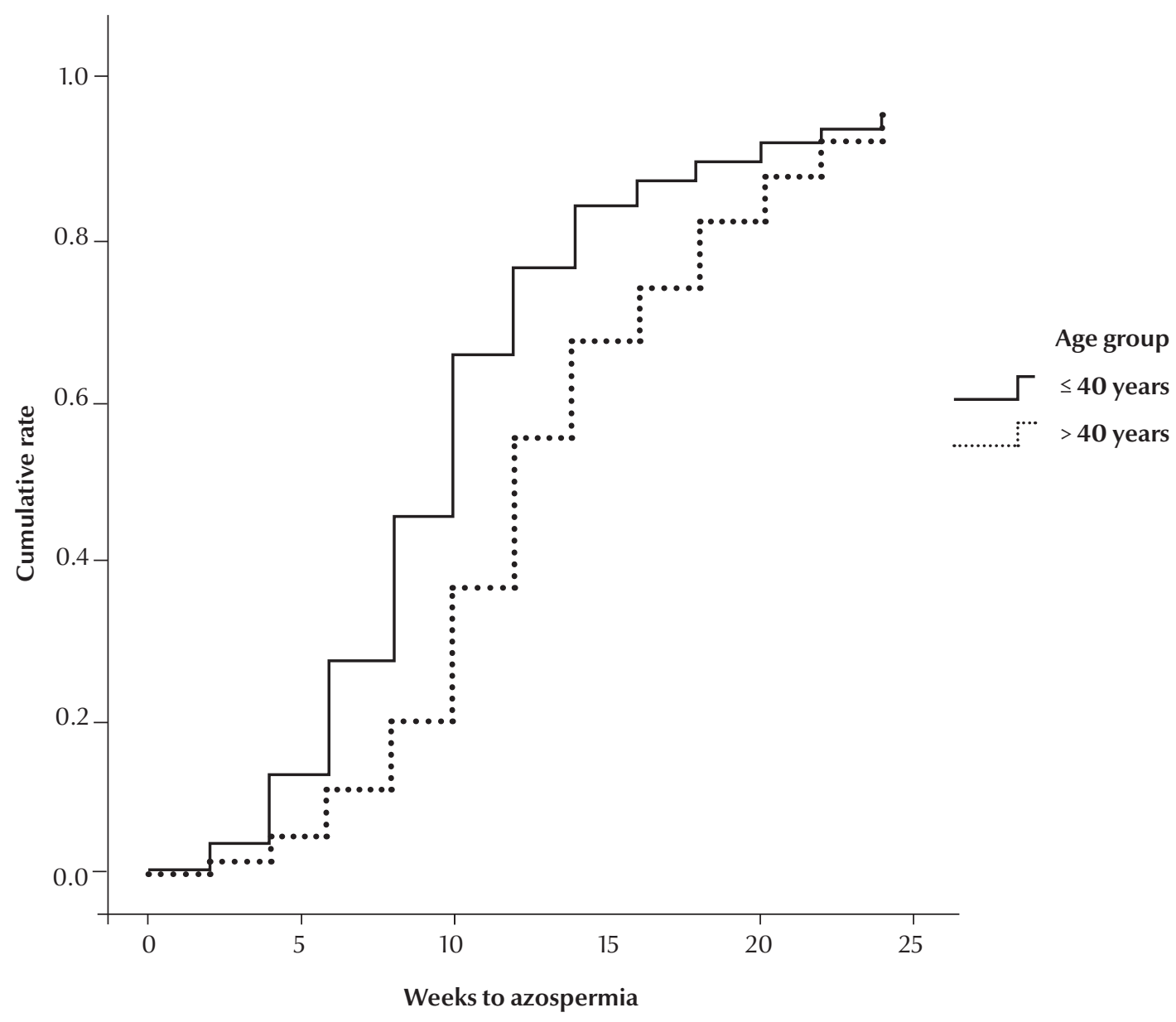

Figure 2 Gross cumulative life table probabilities for azoospermia by age group after no-scalpel vasectomy by ligation and excision with fasacial interposition $(n=334)$

\section{Discussion}

This was a large prospective study which provided detailed information on the success of vasectomy by using the technique of NSV by ligation and excision together with fascial interposition occlusion. Prospectively gathered data with frequent semen analysis are rare in the literature. Our study has shown rapid achievement of azoospermia using this technique, a high cumulative life table rate for azoospermia at the end of week $22(93 / 100)$ and a low proportion of men potentially at risk for fertility at 34 weeks. Younger men reached azoospermia at a faster rate than older men.

Even in developed countries compliance with post-vasectomy follow-up is poor $[9,10]$. In our study only $12.1 \%$ of enrolled men never returned for follow-up after vasectomy and were excluded from analysis, and only $9.0 \%$ of those in analysis could be defined as lost to follow-up. Pre- and post-vasectomy counselling may have been a factor in good post-vasectomy follow-up compliance.

The $2.1 \%$ vasectomy failure rate based on semen analysis in this study was lower than the $11.5 \%$ [15], $12.7 \%$ and $5.9 \%$ [17] failure rates from other well-designed studies, but was similar to the commonly cited failure rate of $1 \%$ to $2 \%$. One of the above-mentioned studies was prospective [15] and the other was a large randomized controlled trial [17], but these high rates were the result of vasectomies without fascial interposition. Our rate is even lower than the failure rates reported elsewhere for vasectomies with fascial interposition
[17]. It should be noted that all vasectomies in our study were performed by master trainers with experience of more than 10000 NSVs and other factors such as the experience of the surgeon may be effective in reducing the failure rate of vasectomy, as others have suggested [17].

A broad range of failure rates have been reported for vasectomy, including pregnancy rates of $4.2 \%$ at 3 years in Nepal [18] and $9.5 \%$ at 5 years in China [19]. Our study outcomes were based on semen analysis rather than on pregnancy data, but it is worth noting that, despite the fact that the participants and their wives were young on average, we had no pregnancies reported in the 2-year period of the study except for 1 case of user failure. This prospective study is continuing to gather longer term follow-up data from study participants 
to examine possible later occurrence of pregnancy, as well as long-term complications and the occurrence of rare non-motile sperms.

At 12 weeks after vasectomy, the cumulative probability for azoospermia was 76.0/100 in this study in contrast with 60.0/100 in a study where fascial interposition was not used for vasal occlusion [15]. Therefore, current guidelines of azoospermia at 12 weeks used in low-resource settings such as many NSV centres in the Islamic Republic of Iran, may leave only about $20 \%$ of men at risk for continued fertility. The results of this study are consistent with the recommendation of Barone et al. that 3 months is a better criterion for doing semen analysis [15].

Within 8 weeks of surgery, $13.2 \%$ of the participants reported one or more related adverse events and this is comparable with other well-designed studies $[15,17]$. All of the adverse events were mild and temporary.
There were some limitations to the study. One limitation was that for religious reasons the men preferred to produced semen samples at home by intercourse rather than by masturbation at the health centre. This caused logistic problems in the delivery of samples to the health centre laboratory for examination within 1 hour, especially for men who lived far from the health centre. However, it is important to note that due to active follow-up and efficient systems at the health centre, all semen samples were examined within 1 hour. The lack of a comparison group or randomization of the participants necessitates caution in interpreting the results.

\section{Conclusions}

Our results indicate that NSV by ligation and excision together with fascial interposition may be effective in decreasing the time to azoospermia, increasing the cumulative life table rate for azoospermia and reducing vasectomy failure rates. It appears that current guidelines for when men can begin to rely on vasectomy for contraception based on 12 weeks after vasectomy can replace semen testing when ligation and excision with fascial interposition is performed by an experienced surgeon.

\section{Acknowledgements}

The authors would like to thank the personnel of the clinic and laboratory for their excellent collection of data and all participants for their cooperation in delivering semen specimens.

Financial support of this study was provided by Population and Family Health Department of Ministry of Health and Medical Education, and by the Deputy for Research, Urmia University of Medical Sciences.

\section{References}

1. Haldar $\mathrm{N}$ et al. How reliable is a vasectomy? Long-term followup of vasectomised men. Lancet, 2001, 356:43-44.

2. Sokal D et al.; the Male Sterilization Investigator Team. A comparative study of the no scalpel and standard incision approaches to vasectomy in 5 countries. Journal of Urology, 1999, 162:1621-1625.

3. Belker AM et al. The high rate of noncompliance for postvasectomy semen examination: medical and legal considerations. Journal of Urology, 1990, 144:284-286.

4. Freund M, Davis JE. Disappearance rate of spermatozoa from the ejaculate following vasectomy. Fertility and Sterility, 1969, 20:163-170.

5. Schmidt SS. Vasectomy by section, luminal fulguration and fascial interposition: results from 6248 cases. British Journal of Urology, 1995, 76:373-374.

6. Marwood RP, Beral V. Disappearance of spermatozoa from ejaculate after vasectomy. British Medical Journal, 1979, 1:87.

7. Richardson DW, Aitken RJ, Loudon NB. The functional competence of human spermatozoa recovered after vasectomy. Journal of Reproduction and Fertility, 1984, 70:575-579.

8. Haws JM et al. Clinical aspects of vasectomies performed in the United States in 1995. Urology, 1998, 52:685-691.

9. Bradshaw HD et al. Review of current practice to establish success after vasectomy. British Journal of Surgery, 2001, 88:290-293.

10. Maatman TJ, Aldrin L, Carothers GG. Patient noncompliance after vasectomy. Fertility and Sterility, 1997, 68:552-555.
11. Huezo CM, Carignan CS. Medical and service delivery guidelines for family planning, 2nd ed. London, Stephen Austin and Sons., 1997.

12. Hatcher RA et al. The essentials of contraceptive technology. Baltimore, Maryland, Population Information Program Center for Communicating Programs, 1997.

13. Pollack AE, Barone MA. Male sterilization. In: Sciarra JJ, ed. Gynecology and obstetrics. Volume 6. Chapter 47. Philadelphia, Lippincott Williams \& Wilkins, 2000.

14. Cortes M et al. Results of a pilot study of the time to azoospermia after vasectomy in Mexico City. Contraception, 1997, 56:215-222.

15. Barone MA et al. A prospective study of time and number of ejaculations to azoospermia after vasectomy by ligation and excision. Journal of Urology, 2003, 170:892-896.

16. World Health Organization. WHO laboratory manual for the examination of human semen and sperm-cervical mucus interaction, 3rd ed. Cambridge, Cambridge University Press, 1993.

17. Sokal D et al.; Investigator Study Group. Vasectomy by ligation and excision, with or without fascial interposition: a randomized controlled trial. BMC Medicine, 2004, 2:6.

18. Nazerali $\mathrm{H}$ et al. Vasectomy effectiveness in Nepal: a retrospective study. Contraception, 2003, 67:397-401.

19. Wang D. Contraceptive failure in China. Contraception, 2002, 66:173-178. 